\title{
What Has Functional Magnetic Resonance Imaging Revealed Regarding the Neurobehavioral Correlates of Sleep Deprivation?
}

\author{
Michael WL Chee, MBBS, MRCP(UK) \\ Professor, Cognitive Neuroscience Laboratory, Neuroscience and Behavioral Disorders Program, Duke-NuS Graduate Medical School Singapore
}

\begin{abstract}
A night of total sleep deprivation can result in performance decline in several cognitive domains. Functional magnetic resonance imaging (fMRI) has helped to reveal the neuroanatomical correlates of such changes in attention and memory, as well as in risky decision-making. Studies of sleep deprivation have shed light on the role of sleep in the consolidation of declarative memory, which can be observed in the form of temporally specific changes in hippocampal and neocortical activation. Sleep deprivation can also be thought of as a cognitive 'stress test' that may afford testing of cognitive enhancers.
\end{abstract}

\section{Keywords}

Sleep deprivation, functional magnetic resonance imaging (fMRI), attention, memory, decision-making

Disclosure: This work was supported by the Defense Science and Technology Agency, Singapore (POD0713897) and a STaR Investigatorship.

Received: January 5, 2009 Accepted: May 4, 2009 DOl: 10.17925/USN.2010.05.02.93

Correspondence: Michael WL Chee, MBBS, MRCP(UK), Neuroscience and Behavioral Disorders Program, Duke-NUS Graduate Medical School, Singapore 169857.

E: michael.chee@duke-nus.edu.sg

Total sleep deprivation (SD) for as little as 24 hours can result in measurable decline in cognitive performance. ${ }^{1}$ Beyond experimental settings, changes in attention, memory, and decision-making contribute to increased physician errors while on shift duty, ${ }^{2,3}$ and deficits in vigilant attention can result in fatal transport accidents. ${ }^{4}$ Despite a growing number of editorials and advisories ${ }^{5}$ regarding the health risks of SD, selfimposed denial of sleep has become increasingly prevalent in urban societies. As such, what was formerly of interest primarily to the military has become a global health and safety concern. This has motivated an awakening of research into the mechanisms underlying SD-related cognitive decline. Additionally, it has become of interest to identify individuals susceptible to the effects of SD and to ascertain the appropriateness of countermeasures deployed to reduce functional deficits where SD is unavoidable. Functional magnetic resonance imaging (fMRI) has attracted considerable interest as a tool in these research areas because it complements observing behavior, provides a clearer spatial localization of cognitive effects than electroencephalography (EEG), and can be performed repeatedly without significant safety concerns. The fMRI studies reviewed here pertain to studies examining task-related activation in sleep-deprived individuals and to reports on the delayed effects of sleep or SD on neural activation. The latter set of studies were performed primarily to characterize memory consolidation.

\section{Imaging Task-related Activation During Sleep Deprivation}

Several cognitive domains have been evaluated in the context of 24,35 , and 48 hours of total SD (see Table 1). Chronic partial SD, while more common, is more difficult to study because proper experimental control is difficult and more expensive to realize.

The best-characterized cognitive domain in the setting of SD is probably working memory. ${ }^{6-14}$ Other facets of cognition studied include verbal learning, ${ }^{15,16}$ sustained attention, ${ }^{17-19}$ divided attention, ${ }^{20}$ visuospatial attention, ${ }^{19,21}$ inhibitory function, ${ }^{22}$ decision-making, ${ }^{23,24}$ and emotional responses to pictures ${ }^{25}$ (see Table 1).

Working memory involves temporary storage and manipulation of information. Tasks designed to evaluate working memory have consistently recruited lateral prefrontal ${ }^{26}$ and parietal cortices. ${ }^{27}$ Most of these studies show that SD tends to reduce task-related activation in these regions, with additional involvement of the ventral visual cortex. However, the manner in which SD influences the functional anatomy of working memory has differed somewhat across studies, possibly as a result of cognitive subtask differences, task difficulty, duration of SD, analytical methods used, and inter-subject variation.

Given the variation in findings, one might ask whether fMRI is capable of yielding reproducible results in the setting of SD. This concern was addressed in a study in which healthy volunteers were studied four times: twice under conditions of SD and twice following a normal night of sleep. The study found that parietal activation decline reproducibly correlated with behavioral decline across state ${ }^{14}$ and concurred with behavioral studies that suggest vulnerability to SD to be trait-like..$^{28,29}$ 
Table 1: Recent Functional Neuroimaging Studies Involving Sleep Deprivation

\begin{tabular}{|c|c|}
\hline Cognitive Domain & Reference \\
\hline \multirow[t]{9}{*}{ Working memory } & Bell-McGinty et al., 2004 \\
\hline & Habeck et al., 2004 ${ }^{10}$ \\
\hline & Chee and Choo, 2004 \\
\hline & Choo et al., $2005^{9}$ \\
\hline & Caldwell et al., $2005^{11}$ \\
\hline & Mu et al., 2005 \\
\hline & Mu et al., $2005^{13}$ \\
\hline & Chee et al., $2006^{8}$ \\
\hline & Lim et al., $2007^{14}$ \\
\hline \multirow[t]{5}{*}{ Attention } & Thomas et al., $2000^{17}$ \\
\hline & Drummond et al., $2001^{20}$ \\
\hline & Drummond et al., $2005^{18}$ \\
\hline & Tomasi et al., $2009^{21}$ \\
\hline & Chee et al., $2008^{19}$ \\
\hline \multirow[t]{2}{*}{ Short-term memory } & Chee and Chuah, $2007^{30}$ \\
\hline & Chuah and Chee, $2008^{57}$ \\
\hline Logical reasoning & Drummond et al., $2004^{60}$ \\
\hline Inhibition (go/no-go) & Chuah et al., $2006^{22}$ \\
\hline \multirow[t]{2}{*}{ Risky decision making } & Venkatraman et al., $2007^{23}$ \\
\hline & Venkatraman et al., $2008^{24}$ \\
\hline Emotional processing & Yoo et al., $2007^{25}$ \\
\hline \multirow[t]{2}{*}{ Verbal learning } & Drummond et al., $2000^{15}$ \\
\hline & Drummond et al., $2005^{16}$ \\
\hline
\end{tabular}

A small number of studies have found that the magnitude of task-related activation following a normal night's sleep can predict an individual's resistance to decline in working memory performance following SD, ${ }^{11,12}$ harking to a form of 'cognitive reserve' in SD-resistant individuals. However, more work needs to be undertaken to evaluate the robustness of these data before fMRI can be deployed as a working tool. ${ }^{14}$

\section{The Neural Correlates of Attention Deficits in Sleep Deprivation}

The notion that failure to sustain attention might underlie decrements in short-term memory was mooted by the finding that increased variability in response time declined more reliably across SD test sessions than memory performance. ${ }^{14}$ This hypothesis was tested in a pair of experiments that parametrically varied visual item load and visual shortterm memory load in tests conducted following normal sleep and after SD. As expected, capacity-limited visual short-term memory capacity was compromised in sleep-deprived persons. Intriguingly, however, SDrelated decline in parietal activation occurred well before memory capacity was saturated. ${ }^{30}$ This finding, together with the attenuation of extrastriate activation at low levels of perceptual load, suggested that deficits in visual attention or visual processing make significant contributions to the reduction of memory capacity observed in SD.

Lapses in attention are associated with response slowing. By analyzing cortical responses according to response time, lapses in SD were found to differ from those occurring after normal sleep by showing lesser modulation of fronto-parietal activation and profound reduction in extrastriate activation. ${ }^{19}$ Thus, visual sensory processing appears to be significantly compromised during attentional lapses that occur in SD. At present, however, it remains unclear whether this was primarily a result
Figure 1: 3D Plots Showing the Results of Trial-by-trial Modeling of fMRI Signal Associated with Slower and Faster Responses for a Given Individual
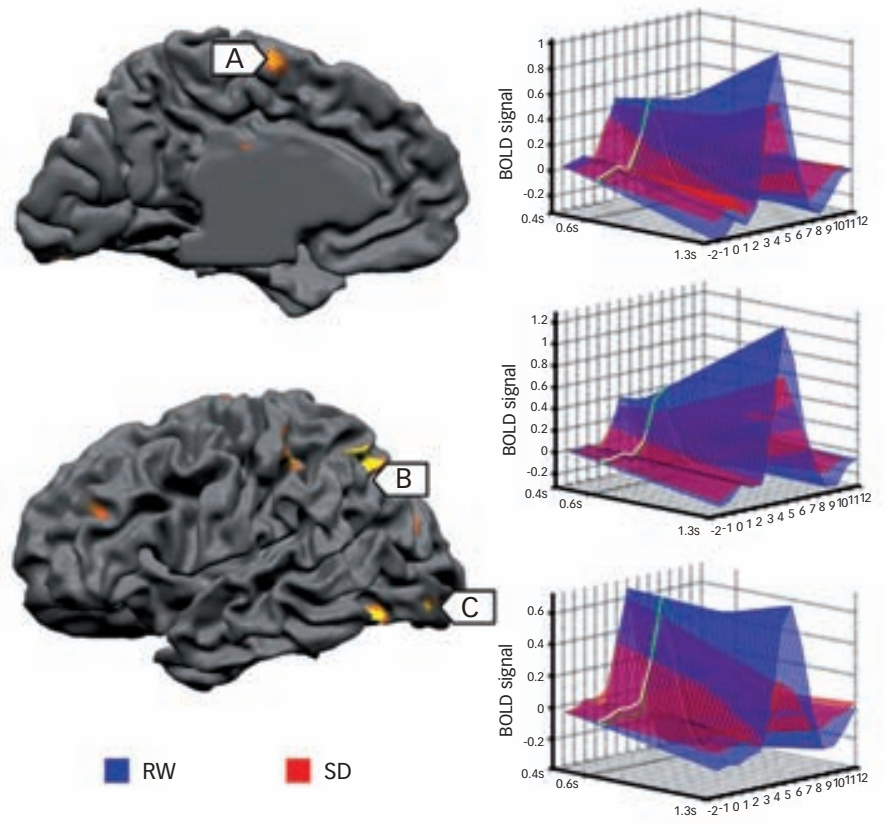

The signal time course at the mean RT is marked in green. A: Medial frontal region; B: Intraparietal sulcus; C: Lateral occipital (extrastriate) cortex. Note that peak signal in the frontoparietal control regions increased with slower responses albeit to a lesser extent during sleep deprivation (SD). By contrast, response slowing was associated with a decrease in extrastriate peak signal during SD. Reproduced with permission from Chee et al., 2008.19

of loss of top-down biasing of visual processing or whether visual cortex was transiently 'shut down' as a consequence of prolonged wakefulness $^{31}$ (see Figure 1). Obliquely related to these findings, it has recently been shown that TMS applied to the left lateral occipital lobe, an area quite consistently affected by SD, can improve working memory in sleep-deprived individuals. ${ }^{32}$

SD results in elevation of task-related activation in the thalamus, ${ }^{19,21,33}$ which may represent a compensation for the overall lower levels of arousal and attenuated fronto-parietal activity that occur when one is sleep-deprived. During lapses in attention, the thalamus shows decrements in activity when under SD but increases in activity after normal sleep. ${ }^{19,21}$ The former may represent some form of sensory gating ${ }^{34}$ whereby the flow of sensory information is attenuated, perhaps to allow a person to slip into sleep undisturbed by the environment.

\section{Decision-making and Emotional Processing}

SD can influence decision-making in a manner that resembles deficits arising from an orbitofrontal lesion. ${ }^{35}$ Persons subjected to extended SD for 49 hours continued to make risky, disadvantageous choices despite sustaining losses ${ }^{36}$ Risky decision-making following even a single night of total SD was found to elicit greater activation of the nucleus accumbens when the riskier of two choices was selected. This signified a higher anticipation of reward without a change in odds. Concurrently, reduced right insula and lateral orbitofrontal activation followed loss trials signaling an attenuated reaction to loss that could impair aversive learning. These gamble-related brain activation changes did not have the behavioral 
accompaniments observed during longer periods of SD. ${ }^{35}$ However, their replication using a different task and a separate cohort ${ }^{24}$ suggests that imaging changes may precede shift in behavior.

To evaluate the relationship between sleep loss and emotion, one study imaged responses to emotionally charged scenes and found increased amygdala responses along with increased brainstem limbic connectivity and reduced amygdalo-medial prefrontal connectivity. ${ }^{25}$ While this observation suggests we might make less circumspect choices when sleep-deprived, more research with different tasks is necessary. For example, slower responding to moral choices ${ }^{37}$ could $^{2}$ indicate an awareness of the need to be less hasty when making decisions when sleep-deprived.

\section{Imaging Studies on Sleep, Memory Consolidation, and Learning}

Consolidation refers to processes that render memories less liable to disruption from interference or disrupting factors. ${ }^{38}$ Consolidation of memory results in improved performance during later testing even without additional practice. ${ }^{39,40}$ Sleep has been shown to benefit nonhippocampal-dependent procedural as well as hippocampal-dependent declarative and spatial memory consolidation (but see reference 41).

Off-line, hippocampal-dependent memory consolidation occurs during slow-wave sleep (SWS). Improvement in the speed of maze navigation following a night of post-learning sleep correlated positively with increases in right hippocampal blood flow during SWS (this was a positron-emission tomography [PET] study). This finding was specific to learning and task in that the positive correlation found with maze training was absent if volunteers learned a serial reaction time task or slept without prior maze training. ${ }^{42}$ Speeded replay of spatial memories acquired during wakefulness has been reported in the hippocampus of sleeping rats, ${ }^{43,44}$ and may explain the increased hippocampal blood flow observed in human studies.

The specificity of the link between hippocampal reactivation during SWS and consolidation of spatial memories was further explored in an elegant set of experiments that associated an odor cue with either a hippocampal-dependent spatial memory task or a non-hippocampaldependent procedural task while the volunteer was awake. Re-exposure to the odor during SWS improved performance in hippocampaldependent but not hippocampal-independent tasks. Additionally, odor re-exposure was ineffective during rapid eye movement (REM) sleep, during wakefulness, or when the odor was omitted at encoding. ${ }^{45}$

Following learning, the circuits recruited during successful task performance change. These transitions in the neural substrates supporting performance may result in less effortful, more automatic responses over time. For example, implicit learning of oculomotor movement sequences elicited greater mid-hippocampal and caudate activation in fast learners, but not in slow learners. ${ }^{46}$ Overnight, there was higher activation in the posterior hippocampus and ventral putamen in fast learners, which correlated with the extent of behavioral improvement.

SD can interfere with this transition and this been demonstrated in hippocampal-independent as well as hippocampal-dependent learning

\section{Figure 2: Sleep Transforms the Cerebral Trace of} Declarative Memory
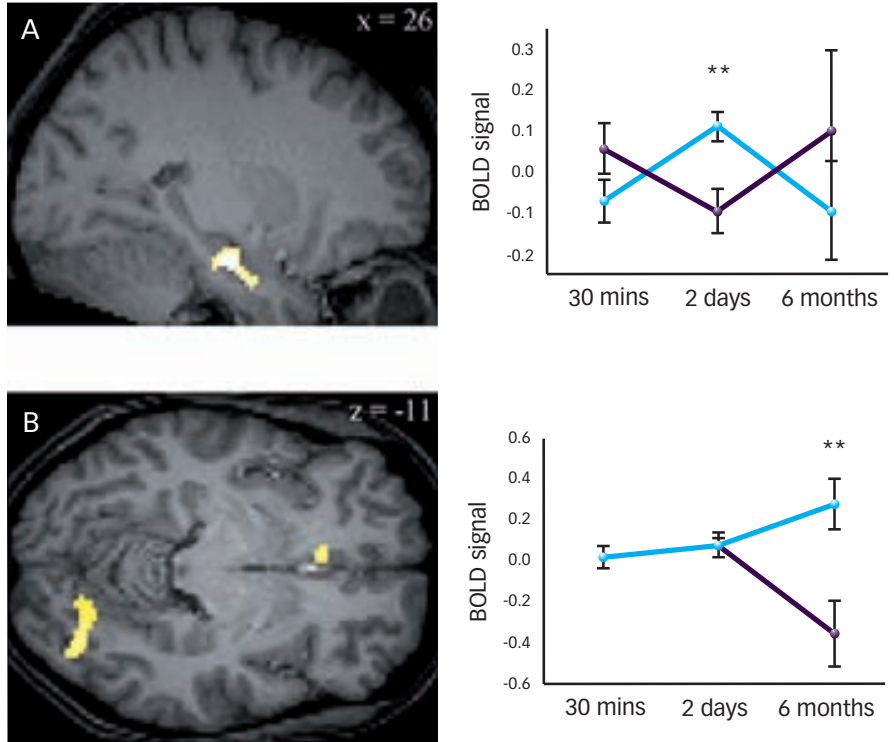

A: Changes in hippocampal activity during correct word recall after a normal night's sleep and after sleep deprivation (SD). Between the immediate (30-minute) and two-day delayed recall sessions, hippocampal involvement in correct word recall increased significantly, but only when subjects slept during the first night after learning. The line plots show hippocampal activity across the whole six-month retention interval. In the sleep condition (blue), activity increased from 30 minutes to two days, whereas no significant changes could be found when subjects were sleepdeprived the night after learning (purple). At the six-month session, hippocampal activity diminished below threshold. B: Differences in medial prefrontal cortex (MPFC) and occipital cortex activity during the six-month retest session for correctly recalled words learned after a normal night's sleep and after SD. Correct word recall after six months activated the MPFC and the occipital cortex more strongly in the sleep condition than in the SD condition. The line plots show MPFC activity across the whole six-month retention interval. The difference in brain activity in the MPFC developed mainly during the interval between the two-day and six-month recall sessions. Note the increase in MPFC activity for words from the sleep condition over the sixmonth period (blue) and a marked drop in MPFC activity for words from the SD condition (purple) during the six-month session. Source: Gais et al., 2007.50

systems. After learning a sequence of finger movements, individuals who slept performed more quickly and showed less activation of prefrontal and premotor areas during retrieval. They also showed greater basal ganglia and parietal activation compared with those who were sleep-deprived. ${ }^{47}$

The consolidation of hippocampal-neocortical-dependent spatial memory associated with maze navigation illustrates how structures initially involved in supporting performance become less relevant over time as different structures come to support optimal performance as a result of consolidation. Whereas hippocampal circuits featured prominently in initial learning and consolidation, volunteers who were allowed to sleep after training showed relatively greater activation of striatum-based circuits compared with similarly trained but sleepdeprived subjects. ${ }^{48}$

Along similar lines, memory circuits associated with declarative memory evidenced striking time-dependent, plastic changes with memory consolidation. Over the course of one to six months, successfully memorized pictures ${ }^{49}$ and word $^{50}$ elicited lower hippocampal activation together with increased medial prefrontal neocortical activation. In the former study, picture recognition at test correlated with hippocampal 
activation the first couple of days after learning but after one and three months, recognition memory became correlated with medial prefrontal and not hippocampal activation. ${ }^{49}$ Critically, in the second experiment involving words, only volunteers who slept evidenced significant 'systems level consolidation' of memory as evidenced by medial prefrontal cortex activation during memory retrieval ${ }^{50}$ (see Figure 2). These human studies show striking agreement with animal studies that showed relatively rapid (weeks to months) development of extrahippocampal support for remote long-term memory. ${ }^{51}$

Interactions between emotional stimuli, sleep, and memory constitute a new and growing area of research. In general, emotional stimuli of any sort are better remembered than neutral stimuli. ${ }^{52}$ Not surprisingly, negative emotional pictures appear to be better remembered than positive pictures after SD. ${ }^{39}$ However, after three days, SD seems to affect memory for negative pictures less than for positive pictures, highlighting the importance of sleep in the consolidation of positive emotional memories. ${ }^{53}$ The differential effect of emotional valence on memory might relate to the routes available for the encoding of each type of picture. Whereas negative pictures recruited the hippocampus and medial prefrontal region in all persons, positive pictures recruited these regions according to how well a given subject remembered these pictures. Critically, relative to non-sleepdeprived subjects, negative pictures in the sleep-deprived group elicited greater amygdalo-cortical activation, suggesting that negative emotional memories could have an alternate route of processing that affords their relatively better preservation. ${ }^{53}$

While these studies support the importance of off-line consolidation or even the development of new insights ${ }^{54}$ into daytime experiences during sleep, it is important to recognize that post-learning plastic changes occur even while we are awake. To demonstrate this, a 'tracking' task was used to evaluate the modulatory effects of spatial and procedural learning on brain activation across multiple scanning sessions. Impressively, the spatial distribution of activation associated with this third, unrelated 'tracking' task was influenced by exposure to the spatial or the procedural task, i.e. in a task-specific fashion. The pattern of brain activation associated with the unrelated task shifted over a twohour period of repeated scans during which volunteers remained awake and did not practice the learned tasks..$^{55}$

\section{Functional Magnetic Resonance Imaging in Intervention and Patient Studies}

Beyond evaluating how SD affects cognition, fMRI can provide useful information concerning the regions affected by drugs administered to preserve or enhance cognitive performance in that setting . ${ }^{56,57}$ This was recently demonstrated in a study that correlated functional imaging and behavioral responses to donepezil administered to sleep-deprived young adults. Cholinergic augmentation selectively benefited individuals vulnerable to the effects of SD whereas resistant subjects showed little improvement or some deterioration, ${ }^{57}$ reminiscent of a similar inverted$u$ response in the dopamine system. ${ }^{58}$ Critically, the drug-induced change in task-driven activation within parieto-occipital regions correlated with behavior modulation. The functional relevance of the imaging findings was buttressed by their correlation with behavioral performance even when volunteers were on placebo.

A non-pharmacological cognitive booster that has been studied with imaging recently is blue light. Monocular exposure to monochromatic blue light at levels below those required to stimulate photopic receptors was found to increase activation of fronto-parietal regions involved in supporting working memory. ${ }^{59}$ The pro-cognitive effects in the setting of dim light were shown to be quick-onset and temporally restricted to the duration of exposure.

\section{Conclusion}

fMRI has proved to be a powerful research tool that has yielded some remarkable insights into the functional neuroanatomy of cognitive decline in SD. In addition, it has enabled us to gain a deeper understanding of the role of sleep in memory consolidation. In the years to come, it is anticipated that these fundamental neuroscience discoveries will be translated in a manner that will enable us to better evaluate and customize countermeasures for a growing number of sleep-deprived persons.
1. Durmer JS, Dinges DF, Semin Neurol, 2005;25:117-29.

2. Landrigan CP, et al., N Eng/ J Med, 2004;351:1838-48.

3. Lockley SW, et al., N Eng/ J Med, 2004;351:1829-37.

4. Horne JA, Reyner LA, BMJ, 1995;310:565-7.

5. Zee PC, Turek FW, Arch Intern Med, 2006;166:1686-8.

6. Bell-MCGinty S, et al., Cereb Cortex, 2004;14:496-502.

7. Chee MW, Choo WC, J Neurosci, 2004;24:4560-67.

8. Chee MW, et al., Neuroimage, 2006;31:419-28.

9. Choo WC, et al., Neuroimage, 2005;25:579-87.

10. Habeck C, et al., Brain Res Cogn Brain Res, 2004;18:306-21.

11. Caldwell JA, et al., Behav Neurosci, 2005;119:694-707.

12. Mu Q, et al., Sleep, 2005;28:433-46.

13. Mu Q, et al., Sleep, 2005;28:55-67.

14. Lim J, et al., Sleep, 2007;30:61-70.

15. Drummond SP, et al., Nature, 2000;403:655-7.

16. Drummond SP, et al., Psychiatry Research: Neuroimaging, 2005;140:211-23.

17. Thomas M, et al., J Sleep Res, 2000:9:335-52.

18. Drummond SP, et al., Sleep, 2005;28:1059-68.

19. Chee MW, et al. I Neurosci, 2008:28:5519-28.

20. Drummond SP, et al., J Sleep Res, 2001;10:85-92.

21. Tomasi D, et al., Cereb Cortex, 2009;19:233-40.

22. Chuah YM, et al., J Neurosci, 2006;26:7156-62.
23. Venkatraman V, et al., Sleep, 2007;30:603-9.

24. Venkatraman V, Chuah YM, Payne JW, et al., Sleep deprivation modulates risky decision-making strategies, Annual meeting of the Society for Neuroscience, Washington DC: Abstract Viewer and Itinerary Planner, 2008, Program No. 715.718.

25. Yoo SS, et al., Curr Biol, 2007;17:R877-878.

26. Curtis CE, D'Esposito M, Trends Cogn Sci, 2003;7:415-23.

27. Pessoa L, et al., Neuron, 2002;35:975-87.

28. Leproult R, et al., Am J Physiol Regul Integr Comp Physiol, 2003;284:R280-290.

29. Van Dongen HP, et al., Sleep, 2004:27:423-33.

30. Chee MW, Chuah YM, Proc Natl Acad Sci U S A, 2007;104:9487-92.

31. Pigarev IN, et al., Neuroreport, 1997;8:2557-60.

32. Luber B, et al., Cereb Cortex, 2008;18(9):2077-85

33. Portas CM, et al., J Neurosci, 1998:18:8979-89.

34. Datta S, Maclean RR, Neurosci Biobehav Rev, 2007;31:775-824

35. Killgore WD, et al., I Sleep Res, 2006;15:7-13

36. Harrison Y, Horne JA, J Exp Psychol Appl, 2000;6:236-49.

37. Killgore WD, et al., Sleep, 2007;30:345-52.

38. McGaugh JL, Science, 2000;287:248-51.

39. Walker MP, Stickgold R, Annu Rev Psychol, 2006;57:139-66.

40. Born J, et al., Neuroscientist, 2006;12:410-24.
41. Vertes RP, Eastman KE, Behav Brain Sci, 2000;23:867-76, discussion 904-1121.

42. Peigneux P, et al., Neuron, 2004;44:535-45.

43. Euston DR, et al., Science, 2007;318:1147-50.

44. Louie K, Wilson MA, Neuron, 2001;29:145-56.

45. Rasch B, et al., science, 2007;315:1426-9.

46. Albouy G, et al., Neuron, 2008;58:261-72.

47. Fischer S, et al., J Neurosci, 2005;25:11248-55.

48. Orban P, et al., Proc Natl Acad Sci U S A, 2006;103:7124-9.

49. Takashima A, et al., Proc Natl Acad Sci U S A, 2006;103: $756-61$.

50. Gais S, et al., Proc Natl Acad Sci U S A, 2007;104:18778-83.

51. Frankland PW, Bontempi B, Proc Natl Acad Sci U S A, 2006;103:509-10.

52. Cahill L, McGaugh JL, Trends Neurosci, 1998;21:294-9.

53. Sterpenich V, et al., PLOS Biol, 2007;5:e282.

54. Wagner U, et al., Nature, 2004;427:352-5.

55. Peigneux $\mathrm{P}$, et al., PLOS Biol, 2006;4:e100.

56. Thomas RJ, Kwong K, Sleep, 2006;29:1471-81.

57. Chuah LY, Chee MW, J Neurosci, 2008;28:11369-77.

58. Mattay VS, et al., Proc Natl Acad Sci U S A, 2003;100:6186-91.

59. Vandewalle G, et al.,Cereb Cortex, 2007;17:2788-95.

60. Drummond SP, et al., Sleep, 2004;27:445-51. 


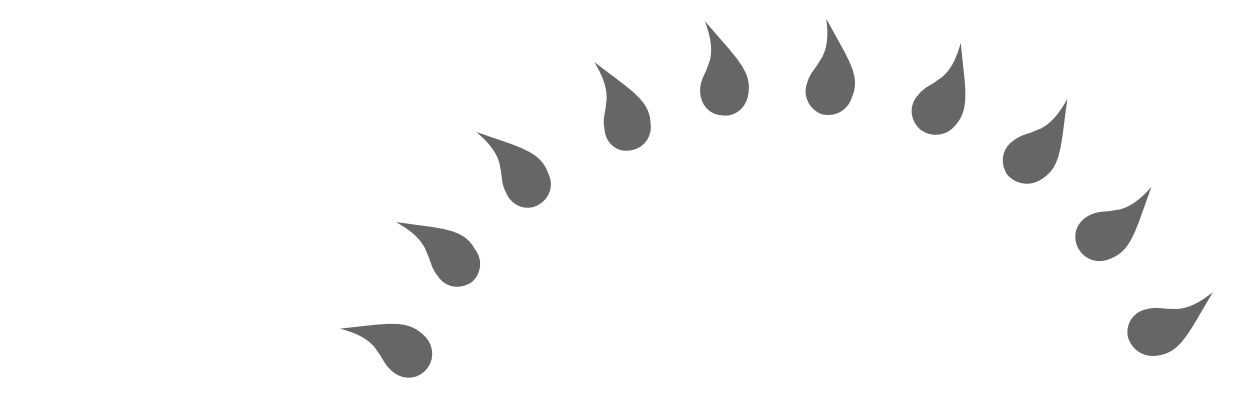

\section{Education, Support and Advocacy}

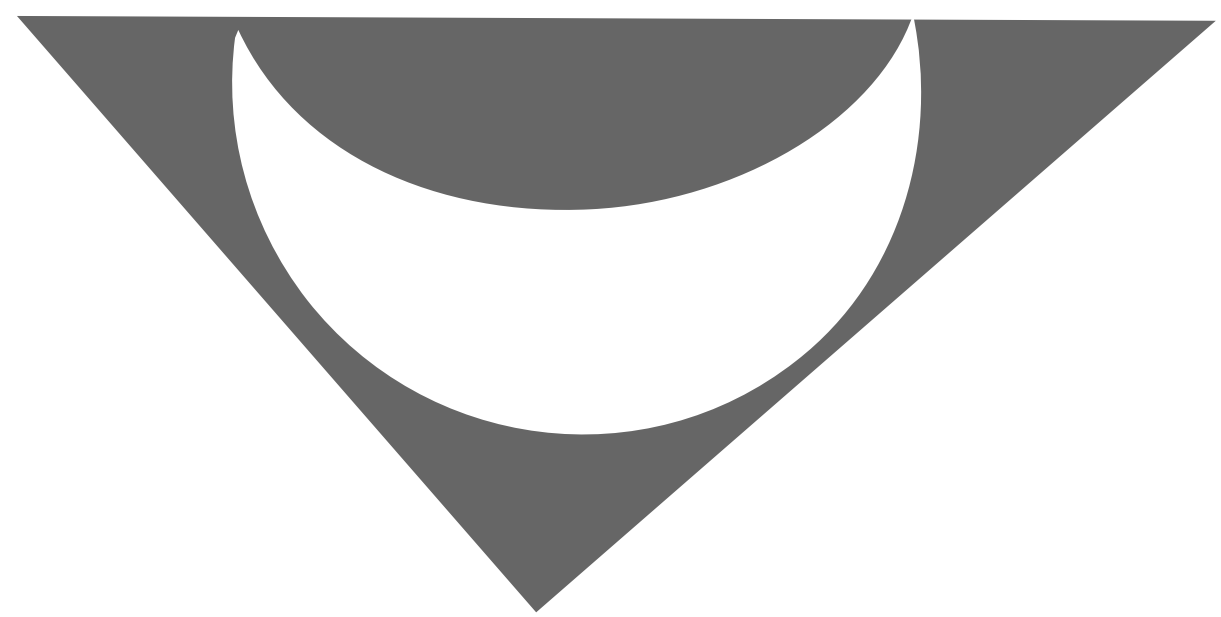

For more than 15 years,

the American Sleep Apnea Association and its A.W.A.K.E. Network of support groups

have provided education, support and advocacy

to those diagnosed and living with Sleep Apnea.

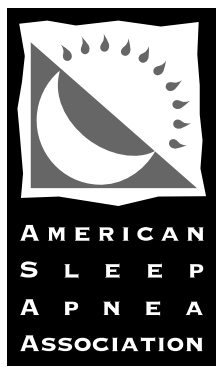

For additional information:

visit: www.sleepapnea.org

email: asaa@sleepapnea.org

write: American Sleep Apnea Association 6856 Eastern Avenue, NW, Ste. 203

Washington, DC 20012

202-293-3650 / 202-293-3656 (fax) 


\section{touch B R I E F I N G S}

Saffron House

6-10 Kirby Street

London

EC1N 8TS

EDITORIAL

Tel: +44 (0) 2074525232

Fax: +44 (0) 2074525050

SALES

Tel: +44 (0) 2074525361

Fax: +44 (0) 2074525606

E-mail: info@touchbriefings.com www.touchbriefings.com

ISSN: 1758-1435 